\title{
Asymptomatic COVID-19 in a Kidney Transplant Recipient
}

\author{
Gizem Kumru Şahin ${ }^{1}$, Pınar Yürük Atasoy ${ }^{2}$ \\ ${ }^{1}$ Ministry of Health Van Education Research Hospital, Department of Nephrology, Van, Turkey \\ ${ }^{2}$ Ministry of Health Van Education Research Hospital, Department of Infectious Diseases, Van, Turkey
}

\section{ABSTRACT}

In December 2019, a novel Coronavirus has been reported to cause wide spectrum of diseases in human and the disease, called Coronavirus Disease 2019, has become an outbreak all around the world with high mortality rate. The disease can present atypically and develop severe complications in immunocompromised solid organ transplantation recipients. Herein we report a kidney transplant patient infected with SARS-CoV-2 infection and presented with an asymptomatic disease despite immunosuppression and comorbidities. Our report shows the importance of social isolation, contact-tracing program and early diagnosis of infection, especially in these high-risk populations. $J$ Microbiol Infect Dis 2020; 8(3):173-176.

Keywords: COVID-19, kidney transplantation, social isolation

\section{INTRODUCTION}

Coronaviruses are a large family of enveloped RNA viruses, some of which are pathogenic to humans and cause wide spectrum of diseases varying from mild infections, such as common cold, to more severe manifestations, such as Middle East Respiratory Syndrome (MERS) and Severe Acute Respiratory Syndrome (SARS) [1]. In December 2019, a series of unexplained viral pneumonia cases have been reported in Wuhan city, Hubei province, China and a novel Coronavirus (2019-nCoV) was identified [2]. Coronavirus Disease 2019 (COVID-19), caused by officially named severe Acute Respiratory Syndrome Coronavirus 2 (SARS-CoV-2), turned into a pandemic spreading all over the world, as on May 13, 2020, the number of patients diagnosed with COVID-19 reached 4088848 and there were 283153 deaths $(6.9 \%$ mortality rate) in more than 200 countries [3]. Considering human-to-human transmission has occurred among close contacts and families with high basic reproductive number (R0), the control of the outbreak has become complicated [4]. COVID-19 has many clinical manifestations within a wide range, including asymptomatic infection, mild upper respiratory tract disease with fever, cough and myalgia, and severe viral pneumonia with respiratory failure [2]. Advanced age and presence of any coexisting illness, such as hypertension, diabetes, coronary heart disease, and chronic obstructive pulmonary disease, are associated with severe disease and mortality $[5,6]$. As immunocompromised patients often present atypical presentations of viral diseases, the clinical features, management and outcomes of COVID-19 in solid organ transplantation (SOT) recipients have not been well-defined yet. Herein, we present a case of COVID-19 in a kidney transplantation (KTx) recipient, which was presented with asymptomatic infection despite coexisting comorbidities and diagnosed with contacttracing.

\section{CASE REPORT}

A 45-year-old man with end-stage renal disease due to nephrolithiasis had undergone 2 nd livingdonor KTx from his wife in 2017. The previous KTx was performed preemptively from his mother in 2005, but during hepatitis $C$ antiviral treatment (ombitasvir/paritaprevir/ritonavir and dasabuvir) the patient had experienced rejection causing graft loss in 2017. Following the 2nd transplant, he was on triple maintenance immunosuppressive therapy with oral tacrolimus (Tac, $1 \mathrm{mg}$ BID), mycophenolic acid (MPA, 720 $\mathrm{mg}, \mathrm{BID}$ ) and prednisolone (Pred, 5mg QD) and has been regularly followed up with serum creatinine $(\mathrm{sCr})$ of $1.29-1.46 \mathrm{mg} / \mathrm{dl}$, estimated glomerular filtration rate (eGFR) of 57-66

Correspondence: Dr. Gizem Kumru Sahin, Ministry of Health Van Education Research Hospital, Department of Nephrology, Van, Turkey 
$\mathrm{mL} / \mathrm{min} / 1.73 \mathrm{~m}^{2}$, Tac trough level of $6-8 \mathrm{ng} / \mathrm{mL}$ and no proteinuria. In addition, the patient also has a history of diabetes, which is well controlled with insulin treatment.

After a patient was diagnosed with COVID-19 on April 06, 2020, contact-tracing was performed with oropharyngeal and nasopharyngeal swabbing in the village of 98 houses and 576 inhabitants where he lived. Our patient's swab tests were reported to be positive for SARSCoV-2 RT-PCR on April 17. It was determined that the first patient and our patient were relatives, and as they were not paying attention to social isolation and infection prevention rules, they have contacted with each other. The village was quarantined on April 18, after 16 people in total were reported to have positive SARS-CoVRNA tests. Due to local culture, household visits were frequent and up to 10 people were living together in households. All patients, including ours, were hospitalized and isolated in the quarantine hospital to reduce the spread of the disease in the village.

At first evaluation, our patient had no symptoms with unremarkable physical examination, including breath sounds on chest auscultation. He was normothermic $\left(36.4{ }^{\circ} \mathrm{C}\right)$, with respiratory rate 14 breaths $/ \mathrm{min}$, heart rate 72 beats $/ \mathrm{min}$, blood pressure $130 / 80 \mathrm{mmHg}$ and oxygen saturation $96 \%$ on room air. The blood examination revealed a white blood cells count of $9,110 \times 10^{3} / \mu \mathrm{L}$, lymphocytes $1,330 \times 10^{3} / \mu \mathrm{L}, \mathrm{C}$ reactive protein $2.3 \mathrm{mmol} / \mathrm{L}, \mathrm{sCr} 1.29 \mathrm{mg} / \mathrm{dL}$; eGFR $66 \mathrm{ml} / \mathrm{min} / 1.73 \mathrm{~m}^{2}$ and Tac level was 7.79 $\mathrm{ng} / \mathrm{ml}$ (Table-1). Alanine aminotransferase (ALT: $47.1 \mathrm{U} / \mathrm{L}$ ) and lactate dehydrogenase (LDH: 265 $\mathrm{U} / \mathrm{L})$ were slightly elevated. Considering his immunocompromised status, he was admitted to the hospital for close monitoring and further testing. No pathological findings suggesting viral pneumonia were reported on chest X-Ray and computed tomography (CT) scan. According to the local treatment guideline, oral hydroxychloroquine (200 mg BID) was initiated and ceftriaxone (1g BID) was added empirically for secondary bacterial infections. His swab tests were reported to be negative for influenza $\mathrm{A} / \mathrm{B}$ and respiratory syncytial virus. Antiproliferative agent was discontinued and his immunosuppressive treatment was maintained with Pred (20 mg QD) and Tac with targeted trough level between 4 and $6 \mathrm{ng} / \mathrm{ml}$.
He reported no high fevers or additional symptoms and his vital signs and renal graft function were stable during hospitalization. Chest CT scan was repeated in the first week and showed no pathological changes. After two negative SARS-CoV-2 RNA tests, the patient was discharged from hospital on April 28, 2020 (hospital day 12) with isolation instructions for home. We rearranged triple maintenance immunosuppressive treatment to their full preillness dosage levels at discharge.

\section{DISCUSSION}

COVID-19 is an easily transmitted respiratory disease caused by SARS-CoV-2. In Turkey, the first case was diagnosed on March 13, 2020 and to date 143114 confirmed cases have been diagnosed [7]. The clinical manifestation of COVID-19 can vary from an asymptomatic disease to acute respiratory distress syndrome requiring intubation and mechanical ventilation. Respiratory virus infections, including Coronaviruses, in SOT recipients may present with mild or atypical symptoms and have higher incidence of infectious complications compared with immunocompetent hosts [8]. Data are limited to understand the clinical course and management KTx patients with COVID-19. Fever, cough and myalgias have been reported as common symptoms of COVID-19 in SOT recipients, similar with general population [911]. As the disease can manifest atypically, all SOT patients presenting with fever or any respiratory or other viral symptoms should be tested for COVID-19.

Close contacts, especially among household members, is a major driver of the outbreak in the community. Of the 262 COVID-19 cases in Beijing, $116(60.4 \%)$ had close contact with confirmed cases and 133 (50.8\%) were family cluster cases [12]. Our patient was a member of a family in which 7 patients were also diagnosed COVID-19. Social isolation is the mainstay to control SARS-CoV-2 infection until vaccination and novel treatments for COVID-19 are available. Low compliance to isolation rules can be an indication of hospitalization for people with positive SARS-CoV-2 RNA test.

Despite no therapies have been shown effective for COVID-19 to date, immunosuppressive therapy was arranged including antimetabolite and/or Tacrolimus discontinuation and treatment 
such as hydroxychloroquine, antiviral therapy, empirical antibacterial, dexamethasone and/or tocilizumab were applied according to the clinical status of patients in recent case series and reports $[9,10,13]$. In some clinically stable and not critically ill patients no Table-1. Laboratory data on admission and follow-up. immunosuppression withdrawal was made and patients recovered successfully. Under the scope of current literature, we only discontinued MPA and made dose management for other immunosuppressive medications in our asymptomatic and clinically stable patient.

\begin{tabular}{|c|c|c|c|c|}
\hline Measure & Reference Range & At admission & Day 7 & At discharge \\
\hline WBC $\left(10^{9} / \mathrm{L}\right)$ & $4-10$ & 9.11 & 8.28 & 7.83 \\
\hline $\operatorname{RBC}\left(10^{12} / L\right)$ & $3.5-5.5$ & 5.61 & 5.51 & 5.48 \\
\hline Hemoglobin (g/dL) & $11-16$ & 16.4 & 15.7 & 15.6 \\
\hline PLT (103/uL) & $100-400$ & 187 & 201 & 198 \\
\hline NEUT $\left(10^{9} / \mathrm{L}\right)$ & $2-7$ & 7.3 & 6.06 & 5.72 \\
\hline $\operatorname{LYM}\left(10^{9} / \mathrm{L}\right)$ & $0.8-4$ & 1.33 & 1.66 & 1.45 \\
\hline CRP (mg/L) & $0-8$ & 2,3 & 0.7 & 0.37 \\
\hline D-dimer (ng/ml) & $0-240$ & 225 & 121 & 93 \\
\hline Fibrinogen (mg/dl) & $238-498$ & 241 & 219 & 244 \\
\hline Prothrombin time (sec.) & $10.2-13.9$ & 12 & 11.7 & 11.9 \\
\hline Blood urea (mg/dL) & $0-50$ & 28 & 22.7 & 35.5 \\
\hline $\mathrm{SCr}(\mathrm{mg} / \mathrm{dL})$ & $0.1-1.2$ & 1.29 & 1.38 & 1.27 \\
\hline eGFR (mL/dk/1.73m²) & $>90$ & 66.98 & 60.88 & 67.31 \\
\hline Sodium (mmol/L) & $135-146$ & 141 & 138 & 137 \\
\hline Potassium (mmol/L) & $3.5-5.1$ & 4.99 & 4.53 & 3.86 \\
\hline Calcium (mg/dL) & $8.2-10.5$ & 9.5 & 9.39 & 9.14 \\
\hline $\operatorname{ALT}(\mathrm{U} / \mathrm{L})$ & $0-40$ & 47.1 & 34.0 & 30.8 \\
\hline AST (U/L) & $0-37$ & 30.3 & 19.4 & 16.1 \\
\hline LDH (U/L) & $120-240$ & 265 & - & 211 \\
\hline Albumin (g/L) & $3.5-5$ & 4.38 & 4.74 & 4.31 \\
\hline Total bilirubin (mg/dL) & $0-1.1$ & 0.3 & 0.3 & 0.2 \\
\hline Urine glucose & Negative & Negative & - & - \\
\hline Urine protein & Negative & Negative & - & - \\
\hline Urine gravity & $1.005-1.025$ & 1.005 & - & - \\
\hline Tacrolimus (ng/mL) & $5-20$ & 7.79 & 4.80 & - \\
\hline SARS-CoV-2 RNA & Negative & Positive & Positive & Negative \\
\hline
\end{tabular}

Abbreviations: ALT, alanine aminotransferase; AST, aspartate aminotransferase; CRP, C-reactive protein; eGFR, estimated glomerular filtration rate, based on CKD-EPI creatinine 2009 equation method; LDH, lactate dehydrogenase; LYM, lymphocyte; NEUT, neutrophil; PLT, platelet; SARS-CoV-2, severe acute respiratory sydrome coronovirus 2 SCr, serum creatinine; WBC, white blood cell.

Our patient experienced mild form of the disease and did not require any specific treatment or intensive care during the disease. Large-scale studies are needed to establish clinical 
manifestations, risk factors and outcome of COVID-19 in SOT patients. There are some case reports that established the successful management of disease without any progression similar to our patient [13], otherwise Huang et al. reported that the possible poor outcome in SOT recipients [14]. An Italian study on $20 \mathrm{KTx}$ patients with COVID-19 demonstrated that $30 \%$ of patients developed acute kidney injury, $20 \%$ of patients required intensive care and $25 \%$ of the patients died despite an aggressive approach [9]. Besides, Akalin et al. reported a very high early mortality rate $(28 \%)$ compared with general population (1\%-6\%) [10]. Considering the possible poor outcome of disease, close monitoring and early and aggressive management is crucial for COVID-19 KTx patients.

\section{Conclusion}

We presented a KTx patient with asymptomatic SARS-CoV-2 infection within a familial cluster. Social isolation and education for infection prevention rules are essential to avoid spread of disease in this high-risk population. Accurate and early diagnosis of COVID-19 with contacttracing program is important to make interventions on time and obtain good outcome.

\section{ACKNOWLEDGMENTS}

Conflict of interest: The authors declare no personal or financial conflict of interest.

Financial Disclosure: No financial support was received.

\section{REFERENCES}

1. Su S, Wong G, Shi W, et al. Epidemiology, Genetic Recombination, and Pathogenesis of Coronaviruses. Trends Microbiol 2016; 24:490-502.

2. Huang $C$, Wang $Y$, Li X, et al. Clinical features of patients infected with 2019 novel coronavirus in Wuhan, China. Lancet 2020; 395:497-506.

3. World Health Organization. Coronavirus disease 2019 (COVID-19) Situation Report - 113 [Internet]. [cited 2020 May 13]. Available from: https://www.who.int/docs/default-

source/coronaviruse/situation-reports/20200512

covid-19-sitrep-113.pdf?sfvrsn=feac3b6d_2

4. Li Q, Guan X, Wu P, et al. Early Transmission Dynamics in Wuhan, China, of Novel CoronavirusInfected Pneumonia. N Engl J Med. 2020; 382:11991207.
5. Wang D, Hu B, Hu C, et al. Clinical Characteristics of 138 Hospitalized Patients with 2019 Novel Coronavirus-Infected Pneumonia in Wuhan, China. JAMA - J Am Med Assoc 2020; 323:1061-1069.

6. Zhou F, Yu T, Du R, et al. Clinical course and risk factors for mortality of adult inpatients with COVID-19 in Wuhan, China: a retrospective cohort study. Lancet. Elsevier Ltd; 2020; 395:1054-1062.

7. Republic of Turkey Ministry of Health. Coronovirus disease (COVID-19)- Current situation in Turkey [Internet]. [cited 2020 May 13]. Available from: https://covid19.saglik.gov.tr/

8. Manuel O, Estabrook M. RNA respiratory viral infections in solid organ transplant recipients: Guidelines from the American Society of Transplantation Infectious Diseases Community of Practice. Clin Transplant 2019; 33:e13511.

9. Alberici F, Delbarba E, Manenti C, et al. A single center observational study of the clinical characteristics and short-term outcome of 20 kidney transplant patients admitted for SARS-CoV2 pneumonia. Kidney Int 2020; 97(6):1083-1088.

10. Akalin E, Azzi Y, Bartash R, et al. Covid-19 and Kidney Transplantation. N Engl J Med 2020 Apr 24. [Epub ahead of print].

11. Fernández-Ruiz $M$, Andrés $A$, Loinaz $C$, et al. COVID-19 in solid organ transplant recipients: a single-center case series from Spain. Am J Transplant 2020 Apr 16.[Epub ahead of print].

12. Tian S, Hu N, Lou J, et al. Characteristics of COVID-19 infection in Beijing. J Infect 2020; 80:401406.

13. Arpali E, Akyollu B, Yelken B, Tekin S, Turkmen A, Kocak B. Case Report: A Kidney Transplant Patient with Mild COVID-19. Transpl Infect Dis 2020 22(4):e13296..

14. Huang J, Lin $\mathrm{H}, \mathrm{Wu} \mathrm{Y}$, et al. COVID-19 in posttransplantation patients-report of two cases. Am J Transplant. 2020 Apr 3. [Epub ahead of print]. 